\title{
Occurrence of Unexpected Adverse Reactions to Vaccines in Children with Mastocytosis
}

\author{
(1) Hanna Abuhay, (1) Alicia S. Clark, (1) Melody C. Carter \\ A Laboratory of Allergic Diseases, National Institute of Allergy and Infectious Diseases, National Institutes of Health, Bethesda, MD
}

\begin{abstract}
Aim: Children with cutaneous or systemic mastocytosis may experience severe manifestations of mast cell mediator release including anaphylaxis. The perceived risk for adverse vaccine reactions creates concern among parents and pediatricians regarding modification of the routine vaccine schedule for safety.

Materials and Methods: Using the National Institutes of Health (NIH) Biomedical Translational Research Information System and Clinical Research Information System, we conducted a retrospective chart review of 94 children $\leq 18$ years of age, evaluated at NIH with mastocytosis. Based on the recommended childhood immunization schedule, we estimated that these 94 patients received approximately 2,136 vaccinations. Post vaccination reactions were determined as expected or unexpected according to the centers for disease control (CDC) parameters for vaccine-associated events.

Results: Eighty-four patients (89.4\%) had no reports of moderate-severe post-vaccination reactions. Eleven reactions after vaccination were reported in 10 of 94 patients (10.6\%), of which four patients had unexpected reactions (4.3\%). Unexpected reactions included facial swelling, flushing and exacerbation of skin lesions which are not reported as possible vaccine reactions by the CDC. One patient was treated for anaphylaxis 2 hours post-varicella vaccine administration. Five patients with a history of anaphylaxis and a mean tryptase level of $115 \mathrm{ng} / \mathrm{mL}$ did not report vaccine-induced reactions.

Conclusion: Children with mastocytosis in this study did not experience a higher rate of adverse vaccine reactions compared to the general population. Anaphylaxis to other causes was not a risk factor for an untoward vaccine response. In patients that experience a severe postvaccination reaction such as anaphylaxis, a modified schedule with single vaccine administration is a safer approach.

Keywords: Pediatrics, mastocytosis, vaccines
\end{abstract}

\section{Introduction}

Mastocytosis is a disease of many variants characterized by abnormal aggregates of mast cells in tissues, most commonly the skin, bone marrow, liver, spleen, and lymph nodes (1). The clinical manifestations of disease include flushing, urticaria, pruritus, diarrhea, and abdominal pain (2). Disease onset may occur both in the pediatric and adult populations, with varying prognoses. Mastocytosis is classified into cutaneous and systemic mastocytosis (SM) based on the presence of mast cell infiltrates in the skin and/or extracutaneous organs, respectively. The serum tryptase value is usually a reflection of total mast cell burden and can range from normal $(<11.4 \mathrm{ng} / \mathrm{mL}$ to significangly elevated in cutaneous and systemic disease. SM, which is commonly seen in adult-onset mastocytosis, may also present in the pediatric age group (1). Cutaneous 
mastocytosis is most common in pediatric onset mastocytosis (3). There are three variants of cutaneous mastocytosis: mastocytoma (MTOMA), maculopapular cutaneous mastocytosis (MPCM), and diffuse cutaneous mastocytosis (DCM) (4). Some common non-immunologic triggers of disease symptoms include stress, change in temperature and exercise (2). Vaccines have been reported as possible triggers of symptoms which has created some concerns regarding the vaccination of patients with mastocytosis (5-7)

There have been a limited number of studies exploring the eliciting nature of vaccines resulting in excessive mast cell degranulation in patients with mastocytosis. Anaphylaxis after vaccination in a patient with DCM has been reported (8). There is a recent report of a patient with DCM that experienced blistering and urticaria following the administration of a live viral vaccine, which prompted a recommendation for premedation before live viral vaccines for patients with DCM (9).

In a separate study, there was a report of nine patients with mastocytosis who experienced mast cell activation triggered by vaccines (5). There have also been two reports of the development of a MTOMA at the site of injection $(10,11)$. In another study, the occurrence of an adverse reaction due to the first administration of a hexavalent vaccine was reported in four patients and these included bullous lesions, urticaria and bronchospasm, which was not seen in the comparison control population (6). On this basis, the first administration of a vaccine to a patient with mastocytosis has been recommended to be done under additional clinical supervision (6). Premedication and single component vaccine administration has also been suggested for patients with DCM, as a preventive measure $(6,7)$.

Previous reports of adverse reactions in patients with mastocytosis associated with vaccination did not identify specific components of vaccines responsible for triggering the release of mast cell mediators. With the exception of the hexavalent vaccine in one study, (6) there are no reports of a particular vaccine more commonly associated with adverse reactions in patients with mastocytosis. The rate of post-vaccination reactions in the general population depends on the type of vaccine. Symptoms such as swelling and redness at the site of injection for the diphtheria, tetanus, and pertussis (DTaP), pneumococcal conjugate vaccine (PCV), human papillomavirus (HPV)- Gardasil09, $\mathrm{Td}$, and Tdap vaccines, range from 0.2 to $90 \%$ of all children (12).
Our current study examines the occurence of postvaccination reactions in children with mastocytosis compared to the data available for the general pediatric population. In order to evaluate whether children with mastocytosis exhibit more reactions due to vaccination, we reviewed the relationship between the number, severity and type of reported reactions to vaccines in patients with mastocytosis. In addition, we looked for correlations with onset and variant of the disease, as well as the type of vaccine associated with the reactions.

\section{Materials and Methods}

Using the NIH Biomedical Translational Research Information System and the $\mathrm{NIH}$ Clinical Research Information System, we conducted a retrospective chart review of 94 children evaluated at NIH from 1984 to 2018 with mastocytosis. The age of the patients when vaccine history was deteremined was $\leq 18$ years of age. Informed consent to participate in the IRB-approved and NIH Ethicsapproved protocol (NCT00044122) was obtained from all parents and also children $\geq 6$ years of age. Mastocytosis variant was determined according to the WHO criteria for cutaneous and systemic disease (4). We queried using the search terms "immunizations", "vaccination" and "vaccines".

The total vaccines administered to patients born between 1994 to 2018 obtained from annual visit questionnaire while at $\mathrm{NIH}$, was determined using the centers for disease control (CDC)'s recommended childhood immunization schedule of the patient's date of birth (13). The vaccine count for patients born between 1984 to 1988 and 1989 to 1993 was determined according to the 1984 and 1989 CDC's immunization schedule, respectively. Haemophilus influenzae type b vaccine ( $\mathrm{HbCV} / \mathrm{Hib}$ ) was only counted for patients born after 1989. For patients born in 1989, one administration of HbCV was counted. The third administration of Hib administered at six months was counted for patients born in 1994 and patients born after 2007. Hepatitis B was counted for patients born after 1990. The second administration of measles, mumps and rubella (MMR) was counted for patients born after 1994. The third administration of Hib was counted for patients born in 1994 and patients born after 2007. The first administration of the varicella vaccine was counted for patients born after 1996 and the second administration was counted for patients born after 2007. Rotavirus vaccine was counted for patients born in 1999 and patients born after 2007. PCV was counted for patients born after 2001. Hepatitis A was counted for patients born after 2006. The changes made 
in CDC's recommended age for administration of specific vaccines between 1984-2018 was taken into account for the vaccine count of patients. Newly added vaccines to the immunization schedule were counted for patients that were of age to receive the vaccine by the time it was administered (Supplemental Figure).

Using the CDC reports, we identified possible side effects to vaccinations based on data from the general population and designated these as expected or as unexpected, the latter of which are reactions not listed in the CDC's report of possible mild or moderate side effects for a given vaccine. Common mild symptoms include fever $\left(100^{\circ} \mathrm{F}\right)$, redness or swelling at the injection site, extended swelling beyond the injection site, fussiness, headache or tiredness. Moderate symptoms are those associated with an interference of usual activities and include fever $\left(102{ }^{\circ} \mathrm{F}\right)$, nausea and vomiting. Based on CDC reports, severe adverse allergic reactions are those that require medical attention such as a serious allergic reaction, can be caused by any medication and are estimated to occur at about 1 in a million and thus, classified as unexpected for our study.

\section{Statistical Analysis}

A univariate analysis was used to describe the distribution of mean age in years for each disease group and measure the standard deviation.

\section{Results}

Ninety-four patients with macular papular cutaneous mastocytosis (MPCM) (59\%), DCM (14\%), MTOMA (5\%) and indolent systemic mastocytosis (ISM) (22\%) were estimated

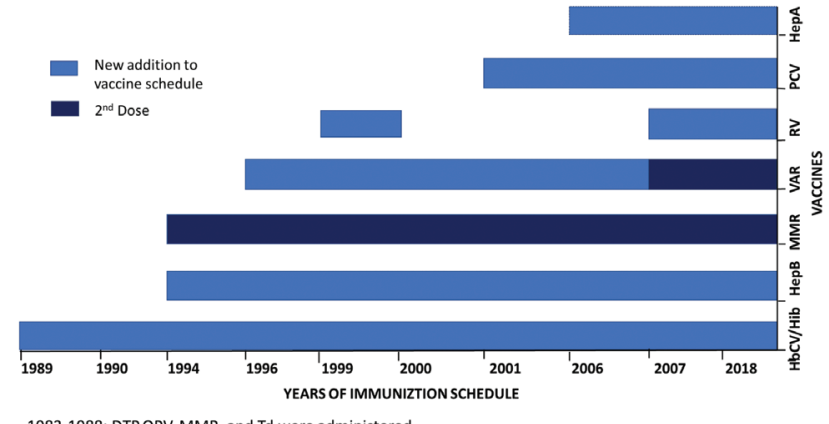

1983-1988: DTP,OPV, MMR, and Td were administered

Supplemental Figure. Changes in CDC Vaccination Schedule 19892018

1983-1988: DTP, OPV, MMR, and Td were administered. Six new vaccines, $\mathrm{HbCV} / \mathrm{Hib}, \mathrm{HepB}, \mathrm{VAR}, \mathrm{RV}, \mathrm{PCV}$, and HepA have been added since 1988. Administration of the RV vaccine was discontinued between 2000 to 2007. A second administration of the MMR and Varicella vaccines was added in 1994 and 2007, respectively.

$\mathrm{RV}$ : Rotavirus vaccine, PCV: Pneumococcal conjugate vaccine, MMR: Measles, mumps and rubella, OPV: Oral poliovirus vaccine, CDC: Centers for disease control to have received a total of 2136 separate administrations of vaccines. When considering adverse vaccine reactions, overall there was no difference in gender distribution amoung those with mild-severe reactions; and patients with MPCM had fewer reported reactions based on total number of vaccines received (Table I). Eighty-five patients (90\%) had the onset of mastocytosis before the age of two years old, of which nine (10\%) patients had reported reactions to vaccines (Table IIA). Nine patients had onset of the disease after two years of age, of which one patient (11\%) reported a reaction to a vaccination. Twenty-three patients at the time of their annual visit and vaccinaiton questionnaire were less than two years old, 15 patients were between the age of 2-6 years old and 56 patients were between the age of 6-18 years old (Table IIB). According to the current

Table I. Demographics of pediatric patients with mastocytosis who received vaccination

\begin{tabular}{|l|l|l|l|l|l|}
\hline Variant & MPCM & DCM & MTOMA & ISM & Total \\
\hline $\begin{array}{l}\text { Number of } \\
\text { patients }\end{array}$ & 55 & 13 & 5 & 21 & 94 \\
\hline Gender F/M & $28 / 27$ & $4 / 9$ & $1 / 4$ & $8 / 13$ & $41 / 53$ \\
\hline $\begin{array}{l}\text { Current } \\
\text { age in years } \\
\text { (mean } \pm \text { SD) }\end{array}$ & $17.13 \pm 7.3$ & $11.27 \pm 7.4$ & $14.40 \pm 7.7$ & $15.54 \pm 7.1$ & $15.84 \pm 7.3$ \\
\hline $\begin{array}{l}\text { Number of } \\
\text { patients with } \\
\text { reported } \\
\text { reactions }\end{array}$ & 4 & 3 & 1 & 2 & 10 \\
\hline
\end{tabular}

MPCM: Maculopapular cutaneous mastocytosis, DCM: Diffuse cutaneous mastocytosis,

MTOMA: Mastocytoma, ISM: Indolent systemic mastocytosis, SD: Standard deviation

\begin{tabular}{|l|l|l|}
\hline \multicolumn{2}{|l|}{ Table IIA. Age of onset of mastocytosis } \\
\hline Age of onset & Before 2 years & After 2 years \\
\hline Number of patients & 85 & 9 \\
\hline $\begin{array}{l}\text { Number of patients with } \\
\text { reactions }\end{array}$ & 9 & 1 \\
\hline
\end{tabular}

Table IIB. Vaccine history related to age

\begin{tabular}{|l|l|l|l|}
\hline Age Group & $\begin{array}{l}\text { Birth } \mathbf{- 2} \\
\text { years }\end{array}$ & $\begin{array}{l}\mathbf{2 - 6} \\
\text { years }\end{array}$ & $\begin{array}{l}\mathbf{6 - 1 8} \\
\text { years }\end{array}$ \\
\hline Number of patients & 23 & 15 & 56 \\
\hline Total Vaccine doses/patient & 23 & 6 & 1 \\
\hline Total Vaccine types/patient & $8 *$ & $5+$ & $1 *$ \\
\hline
\end{tabular}

Various ages are represented in each age range and not reflective of total vaccines administered. * :DTaP, IPV, Hep A, Hep B, Hib/HbCV, MMR, PCV, VAR; †: Hep A DTap, IPV, MMR, VAR; ${ }^{\ddagger}$ : Tdap

DTaP: Diphtheria, tetanus, and pertussis, IPV: Inactivated polio vaccine, MMR:

Measles, mumps and rubella, VAR 
vaccination recommendation, a total of 23 administrations of eight different vaccines are to be given before age 2 years. Between the two groups (2-6 and 6-18 years), a total of seven administrations of six different vaccines are recommended (Table IIB). At the time of data collection, patients through age 6 years (60\%) had received $96 \%$ of the recommended vaccines and thus the data reflects the majority of possible reported events.

Eighty-four (89.4\%) of the 94 patients did not have any moderate-severe reactions to vaccinations. Ten patients were reported to have a total of 11 expected and unexpected reactions associated with vaccination after onset of

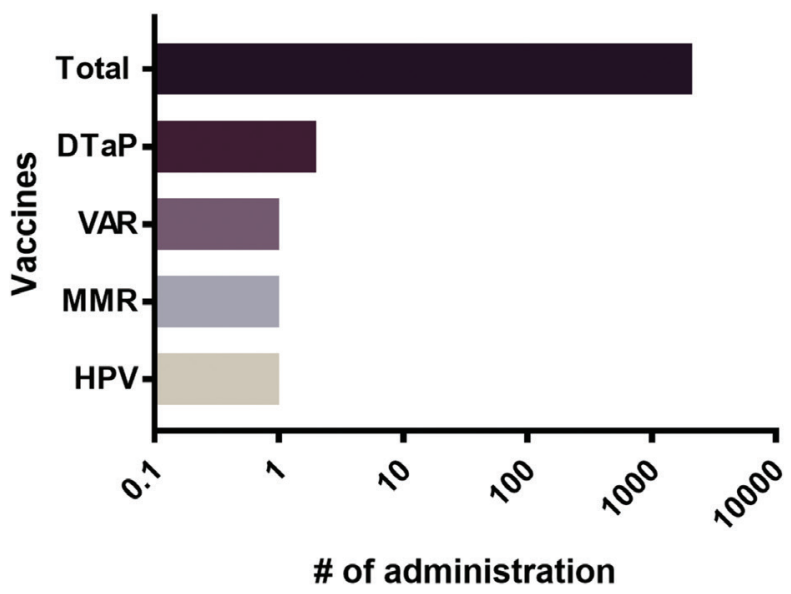

Figure. Total Vaccine administrations and vaccines associated with unexpected reactions (URs)

2136 vaccines were administered to 94 patients (Total bar), of which 4 administrations were associated with URs (remaining bars). In 3 patients with

URs, DTap was associated with one alone; MMR and HPV with DTaP in one and VAR with one. In the fourth patient, the eliciting vaccine was not identified (data not shown), MMR: Measles, mumps and rubella, HPV: Human papillomavirus, DTaP: Diphtheria, tetanus, and pertussis, VAR: mastocytosis. The time of occurrence of a post-vaccination reaction was specified for one reaction associated with anaphylaxis. The mastocytosis variant of the six patients with expected reactions included MPCM, DCM, ISM and MTOMA, with tryptase levels ranging from $3.4-139 \mathrm{ng} /$ $\mathrm{mL}$ (data not shown). The four patients that experienced unexpected vaccine reactions had tryptase values ranging from 5.1-62.9 ng/mL (Table III). Thus, the serum tryptase level was not predictive of an adverse reaction in our cohort.

Four patients (4\%) had reactions that were associated with unexpected symptoms including anaphylaxis, urticaria or exacerbation of skin lesions, flushing, facial swelling and Gl symptoms beyond the common expected side effects enlisted by the CDC, such as fever, redness and swelling at the site of injection (Table III). Four vaccinations (DTaP, MMR, HPV and Varicella) out of the 2136 (0.18\%) administered were reported as responsible for the unexpected reactions (Figure). Patient 1 with MPCM was reported to have experienced flushing, abdominal pain, vomiting, wheezing, dizziness and to have had an anaphylactic episode two hours after receiving the second varicella vaccine. The patient was treated with epinephrine. A second patient (patient 2) with MPCM, experienced eye swelling and flushing with the DTaP vaccine. Similarly, a patient 3 who had ISM experienced facial swelling, flushing and accentuation (swelling) of lesions of MPCM following the administration of HPV, DTaP and MMR. A patient with DCM, patient 4, reported mild flushing and urticaria, but the eliciting vaccine was unknown (Table III). These reactions were unique to patients with mastocytosis and not reported as common adverse reactions in the general population.

There were five other patients with a history of anaphylaxis who did not have any vaccine-induced reactions (Table IV). One of these patients had experienced anaphylaxis

Table III. Clinical data of patients with unexpected reactions to vaccination

\begin{tabular}{|c|c|c|c|c|c|c|}
\hline Patient & $\begin{array}{l}\text { Age of } \\
\text { disease onset }\end{array}$ & $\begin{array}{l}\text { Tryptase level } \\
\text { (ng/mL) }\end{array}$ & Variant & $\begin{array}{l}\text { Reaction inducing } \\
\text { vaccine }\end{array}$ & Reaction & Treatment \\
\hline 1 & Birth & 5.1 & MCPM & varicella & $\begin{array}{l}\text { flushing, abdominal pain, } \\
\text { vomiting, wheezing and } \\
\text { dizziness (anaphylaxis) }\end{array}$ & epinephrine \\
\hline 2 & 5 months & 6.1 & MCPM & DTaP & eye swelling, flushing & $\mathrm{N} / \mathrm{A}$ \\
\hline 3 & 15 years & 62.9 & ISM & $\begin{array}{l}\text { HPV } \\
\text { DTaP } \\
\text { MMR }\end{array}$ & $\begin{array}{l}\text { facial swelling, flushing and } \\
\text { exacerbation of MPCM lesions }\end{array}$ & $N / A$ \\
\hline 4 & Birth & 23.4 & DCM & N/A & mild flushing and urticaria & $\mathrm{N} / \mathrm{A}$ \\
\hline
\end{tabular}




\begin{tabular}{|l|l|l|l|}
\hline \multicolumn{2}{|l|}{ Table IV. Anaphylaxis history } \\
\hline & $\begin{array}{l}\text { Number } \\
\text { of } \\
\text { patients }\end{array}$ & Trigger & $\begin{array}{l}\text { Age at } \\
\text { time of } \\
\text { episode }\end{array}$ \\
\hline $\begin{array}{l}\text { Non-vaccine associated } \\
\text { anaphylaxis }\end{array}$ & 5 & $\begin{array}{l}\text { Idiopathic } \\
\text { anaphylaxis* } \\
\text { Fire Antt }\end{array}$ & $\geq 2$ years \\
\hline $\begin{array}{l}\text { Vaccine associated } \\
\text { anaphylaxis }\end{array}$ & 1 & Varicella vaccine & 5 years \\
\hline $\begin{array}{l}\text { *: One episode each of idiopathic anaphylaxis in four patients; t: Two episodes } \\
\text { of fire ant-induced anaphylaxis in one patient }\end{array}$ \\
\hline
\end{tabular}

to fire ant and the remaining four were diagnosed with idiopathic anaphylaxis. Although previous anaphylaxis to a vaccine is reported to be associated with an increased risk of subsequent severe reactions, anaphylaxis to other causes was not associated with the same increase risk in our study.

\section{Discussion}

The prevalence of vaccine-induced reactions was not greater in our patients with mastocytosis compared to the general population and the unexpected reactions occurred in $4.2 \%$ of our patients. With the exception of anaphylaxis, the unexpected reactions in patients with mastocytosis were illustrative of mast cell-medicated symptoms associated with their primary disease and therefore not reported in the general population. Eighty-nine percent of the patients in this study did not have a history of post-vaccination reactions and only $0.18 \%$ of vaccine administrations were associated with unexpected reactions (Figure). Most pediatric vaccines $(77 \%)$ are administered before the age of two. Since $75 \%$ of our patients are in the age group (>age 2 years) that received most of the recommended vaccines, we feel this data is reflective of a low risk for unexpected postvaccination reactions.

Expected reactions to vaccines are reported in the general population. The mild and moderate symptoms associated with specific vaccines occur at varying prevalence reported as $0.2 \%$ to $90 \%$ depending on the vaccine (12). Only seven patients $(7.4 \%)$ in our cohort had reports of symptoms such as fever, localized rash, swelling and redness at site of injection, which are expected reactions identified by the CDC as commonly reported side effects. Thus, the safety profile in our patients with mastocytosis did not suggest an increased risk of severe reactions to vaccines.

Unexpected reactions were reported in four patients $(4.2 \%)$, of which one patient had a report of second expected reaction as well. Four vaccines, HPV, DTaP, MMR, and Varicella were reported to be associated with these unexpected reactions (Figure). Although the adverse event to the second dose of varicella in patient one was severe enough to require administration of epinephrine, the patient suffered no prolonged or residual side effects and was able to tolerate subsequent vaccines. Anaphylaxis to vaccines reportedly identifies patients as high risk for subsequent vaccinations (14). Vaccine components such as, yeast, egg, gelatin, and latex have been linked to some post-vaccination allergic reactions in the general population (14). Thus, it has been suggested that patients with anaphylaxis to vaccines be evaluated for the responsible allergen as a preventive measure (14). Additionally, the administration of single vaccines has been widely recommended for patients with mastocytosis to include extended post-vaccination medical supervision for 30 minutes to 2 hours $(6,14)$. Based on our experience, we think a post-vaccination observation period, as recommened in previous studies, is a reasonable approach.

Five patients reported episodes of anaphylaxis that were not attributed to any vaccines. These reactions in these patients were noted to have occurred at $\geq 2$ years, after they had received the majority of their vaccines. According to the risk assessment for adverse vaccine reactions published by the European Academy of Allergy and Clinical Immunology (14), patients who have experienced anaphylaxis unrelated to vaccines are not thought to be at higher risk to an adverse vaccine response (14). Our data also supports this observation and these patients do not have to opt out of receiving vaccines.

There was no particular trend with respect to mastocytosis variant or serum tryptase level noted amongst patients with post-vaccination reactions. Patients with extensive cutaneous involvement such as DCM and elevated serum tryptase levels have been reported to be at a higher risk for severe mast cell activation episodes (5). Although patients with DCM represent a smaller number (14\%) in our study, the number of reactions was similar to the patients with MPCM (59\%) (Table 1). It is, however, important to note that the most severe reaction occurred in a patient who would not have been predicted to be at high risk for severe mast cell activation reactions. This patient has MPCM, a tryptase level of $5.1 \mathrm{ng} / \mathrm{mL}$ and no previous adverse reaction to vaccine administration. On the contrary, the five patients with a history of anaphylaxis and a mean tryptase level of $115 \mathrm{ng} / \mathrm{mL}$ had no reports of reactions due to vaccines.

Our study limitations are based on limited sample size for the variant, DCM, MTOMA and ISM and thus a quantitative comparison in the number of reactions was not 
made between variants as a result. The vaccine responsible for the unexpected reaction in patient 4 was not available in our medical chart. In addition, the temporal association between three of the unexpected reactions and vaccine administration was not precisely identified.

\section{Conclusion}

Collectively, our data agrees with the literature and does not indicate patients with mastocytosis to be at an overall greater risk for vaccine-induced reactions. Patients with DCM may be at higher risk for more severe reactions to a variety of triggers including vaccines as noted in several case reports $(5,7,9)$. However, vaccines were not a trigger in several of our patients indicated to be at higher risk for severe mast cell activation episodes. Nonetheless, precaution such as prolonged medical supervision (30 minutes-2 hours) following vaccination should be taken when vaccinating as there are a few reports of patients with unexpected reactions, which can be severe. This is particularly important in patients with a history of a previous expected moderate reaction or an unexpected reaction of any severity.

\section{Acknowledgements}

Dean D Metcalfe, MD - Critical review and editing of the manuscript. Linda Scott and Daly Cantave-coordinated and supervised data collection.

\section{Ethics}

Ethics Committee Approval: National Institutes of Health Ethics- approved protocol (NCT00044122)

Informed Consent: Informed consent to participate in the IRB-approved protocol (NCT00044122) was obtained from all parents and also children $\geq 6$ years of age.

Peer-review: Externally peer-reviewed.

\section{Authorship Contributionss}

Design: A.S.C., H.A., M.C.C., Data Collection or Processing: H.A., Analysis or Interpretation: A.S.C., H.A., M.C.C., Writing: H.A., M.C.C

Conflict of Interest: The authors have no conflicts of interest relevant to this article to disclose.

Financial Disclosure: This work was supported by the Division of Intramural Research, National Institute of Allergy and Infectious Diseases, National Institutes of Health.

\section{References}

1. Valent P, Akin C, Escribano L, et al. Standards and standardization in mastocytosis: Consensus Statements on Diagnostics, Treatment Recommendations and Response Criteria. Eur I Clin Invest 2007;37:435-53.

2. Broesby-Olsen S, Carter M, Kjaer HF, et al. Pediatric Expression of Mast Cell Activation Disorders. Immunol Allergy Clin North Am 2018;38:365-377.

3. Hartmann K, Escribano L, Grattan C, et al. Cutaneous manifestations in patients with mastocytosis: Consensus report of the European Competence Network on Mastocytosis; the American Academy of Allergy, Asthma \& Immunology; and the European Academy of Allergology and Clinical Immunology. I Allergy Clin Immunol 2016;137:35-45.

4. Akin C, Valent P. Diagnostic criteria and classification of mastocytosis in 2014. Immunol Allergy Clin North Am 2014;34:207-18.

5. Alvarez-Twose I, Vañó-Galván S, Sánchez-Muñoz L, et al. Increased serum baseline tryptase levels and extensive skin involvement are predictors for the severity of mast cell activation episodes in children with mastocytosis. Allergy 2012;67:813-21.

6. Parente R, Pucino V, Magliacane $D$, et al. Evaluation of vaccination safety in children with mastocytosis. Pediatr Allergy Immunol 2017;28:93-5.

7. Bankova LG, Walter JE, lyengar SR, Lorenzo ME, Hornick IL, Castells MC. Generalized Bullous Eruption after Routine Vaccination in a Child with Diffuse Cutaneous Mastocytosis. J Allergy Clin Immunol Pract 2013;1:94-6.

8. Brockow K, Jofer C, Behrendt H, Ring J. Anaphylaxis in patients with mastocytosis: a study on history, clinical features and risk factors in 120 patients. Allergy 2008;63:226-32.

9. Gupta M, Akin C, Sanders GM, Chan MP, Ross CW, Castells MC. Blisters, Vaccines, and Mast Cells: A Difficult Case of Diffuse Cutaneous Mastocytosis. The Journal of Allergy and Clinical Immunology: In Practice 2019;7:1370-2.

10. Koh M), Chong WS. Red Plaque After Hepatitis B Vaccination. Pediatr Dermatol 2008;25:381-2.

11. Poulton JK, Kauffman CL, Lutz LL, Sina B. Solitary mastocytoma arising at a hepatitis B vaccination site. Cutis 1999;63:37-40.

12. Center for Disease Control and Prevention. Possible Side-effects from Vaccines. Vol 2019. ed2018.

13. Center for Disease Control and Prevention. Resource Library: Prior Years' Child/Adolescent Immunization Schedules. Vol 20182018.

14. Nilsson L, Brockow K, Alm J, et al. Vaccination and allergy: EAACl position paper, practical aspects. Pediatr Allergy Immunol 2017;28:628-40. 